\title{
The Heliophysics Feature Catalogue, a tool for the study of solar features
}

\author{
Xavier Bonnin ${ }^{1}$, Nicolas Fuller ${ }^{1}$, Christian Renié ${ }^{1}$, Jean \\ Aboudarham $^{1}$, Baptiste Cecconi ${ }^{1}$, Robert D. Bentley ${ }^{2}$ \\ and André Csillaghy ${ }^{3}$ \\ ${ }^{1}$ LESIA, Observatoire de Paris, CNRS, UPMC, Universit Paris-Diderot, 5 place Jules Janssen, \\ 92195 Meudon, France \\ email: xavier.bonnin@obspm.fr email: nicolas.fuller@obspm.fr \\ email: christian.renie@obspm.fr email: jean.aboudarham@obspm.fr \\ email: baptiste.ceconni@obspm.fr \\ ${ }^{2}$ MSSL, University College London, Hombury St. Mary, Dorking, Surrey RH5 6NT, U.K. \\ email: rdb@mssl.ucl.ac.uk \\ ${ }^{3}$ Institute of 4D Technologies, FHNW, Steinackerstrass 5, CH-5210 Windisch, Switzerland \\ email: andre.csillaghy@fhnw.ch
}

\begin{abstract}
The behavior of filaments and prominences during the Solar Cycle is a signature of Sun's activity. It is therefore important to follow their evolution during the cycle, in order to be able to associate it with the various phases of the Solar Cycle as well as with other Solar features or events. The virtual observatory HELIO provides information that can be used for such studies, especially its Heliophysics Feature Catalogue gives a unique access to the description of various features during around one cycle. Features available are: filaments, prominences, photospheric and coronal active regions, coronal radio emission, type III radio bursts, coronal holes and sunspots. Web interfaces allow the user to query data for these features. Useful information can also be shared with other HELIO services, such as Heliophysics Event Catalogue, which provides access to dozens of tables of events such as flares, CMEs, ...
\end{abstract}

Keywords. Solar features, Automatic recognition, Image processing, Database, Data mining, Virtual observatory

\section{Introduction}

In the frame of the European project HELIO (Bentley et al. 2011), funded under European Commission's seventh Framework Program, (Project No. 238969) the Heliophysics Feature Catalogue (HFC) has been growing for the three last years. It is populated using automatic solar and heliospheric features detection codes which have been mainly developed at LESIA (Paris Observatory, France) and TCD (Dublin, Ireland). Currently, the HFC holds descriptions of filaments, prominences, active regions, coronal holes, sunspots, type III radio bursts and radio sources, which are searchable through dedicated user interfaces. From that, it is possible to study the behavior of several kinds of signatures of the solar activity, and possibly look for correlations between them. With the development of tracking capabilities it is also possible to derive events from the observations, like filaments disparitions brusques which are linked to CMEs. The HFC graphical user interface is accessible at: http://voparis-helio.obspm.fr/hfc-gui/, and more information about HELIO can be found at: http://www.helio-vo.eu.

Next Section provides an overview of the detection codes used to populated the HFC. 


\section{Detection Tools}

SoSoFT (Fuller et al. 2005) is dedicated to the segmentation of solar filaments on full Sun $\mathrm{H} \alpha$ images. After some cleaning steps (irregular background intensity, dust lines, etc.), seed pixels of dark areas are obtained using a windowed threshold. The seeds are then grown to catch the whole filament (region growing technique). The segmented filament are described by using morphological operators to retrieve shape and position parameters. TrackFil (Bonnin et al. 2013) is a filament tracking code that uses a curve-matching algorithm to compare the shape of filament skeletons on successive images.

SoSoPro has been developed together with SoSoFT to detect filaments, but on the limb, where they are called prominences. We use CaII K3 images from Meudon Observatory to detect them with a region growing technique. A raster scan of each prominence is then encoded (Run Length Encoding) and stored in the HFC, together with parameters like coordinates, height, etc.

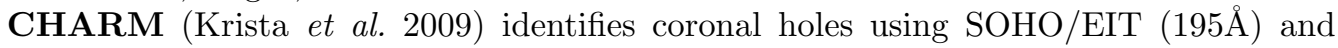
$\mathrm{SOHO} / \mathrm{MDI}$ observations. It uses a histogram-based intensity thresholding technique to detect low intensity regions on EIT and then classifies them as coronal holes or filaments using magnetograms.

NRH2D, developped by Christian Renie, detects and tracks radio sources on 2D images at 150 or $164 \mathrm{Mhz}$ observed by Nancay radioheliograph. A gaussian fit is performed on the source to describe its shape.

SMART (Higgins et al. 2011) algorithm relies on consecutive image differencing to remove both quiet-Sun and transient magnetic features on MDI magnetograms to detect solar active regions. A region-growing technique allows to group flux concentrations into classifiable features. SMART extracts both position and magnetic properties of the features.

RABAT3 (Bonnin et al. 2011) applies a specific Hough transform on a binary image of a dynamical spectrum to determine the type III main direction.

SDOSS is an upgrade of the code developed for EGSO to detect sunspots on SOHO/MDI (MDISS) (Zharkova et al. 2005), which have been superseded by SDO/HMI. The detection is based on the watershed operator and on the Canny edge technique. SDOSS efficiently discriminates umbra and penumbra in a given sunspot.

SPOCA (Barra et al. 2009) uses a multi-channel unsupervised spatially-constrained fuzzy clustering method, which automatically segments solar extreme ultraviolet (EUV) images into regions of interest. It is successfully applied on SOHO/EIT $(195 \AA, 171 \AA)$

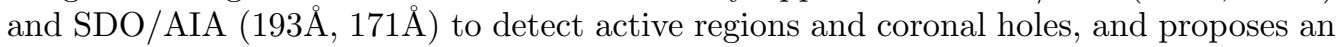
additional algorithm to track features with the solar rotation.

\section{References}

Barra, V., Delouille, V., Kretzchmar, M, \& Hochedez, J.-F. 2009, A\&A, 505, 361

Bentley, R. D., Csillaghy, A., Aboudarham, J., Jacquey, C., Hapgood, M. A., Bocchialini, K., Messerotti, M., Brooke, J., Gallagher, P., Fox, P., Hurlburt, N., Roberts, D. A., \& Duarte, L. 2011, Adv. Sp. Res., 47, 2235

Bonnin, X., Aboudarham, J., Fuller, N., Csillaghy, A., \& Bentley, R. D. 2013, Solar Phys., 283, 49

Bonnin, X., Aboudarham, J., Fuller, N., Renie, C., Perez-Suarez, D., Gallagher, P., Higgins, P., Krista, L., Csillaghy, A., \& Bentley, R. D. 2011, SF2A-2011 Conf. Proceed., 373 
Fuller, N., Aboudarham, J., \& Bentley, R. D. 2005, Solar Phys., 227, 61

Higgins, P., Gallagher, P., McAterr, R., \& Bloomfield, D. 2011, Adv. Sp. Res., 47, 2105

Krista, L. \& Gallagher, P. 2009, Solar Phys., 256, 87

Zharkova, V., Aboudarham, J., Zharkov, S., Ipson, S., Benkhalil, K., \& Fuller, N. 2005, Solar Phys., 228, 361 